CORRECTION

https://doi.org/10.1038/s41586-018-0664-3

\title{
Author Correction: Nearly all the sky is covered by Lyman- $\alpha$ emission around high-redshift galaxies
}

L. Wisotzki, R. Bacon, J. Brinchmann, S. Cantalupo, P. Richter, J. Schaye, K. B. Schmidt, T. Urrutia, P. M. Weilbacher, M. Akhlaghi, N. Bouché, T. Contini, B. Guiderdoni,

E. C. Herenz, H. Inami, J. Kerutt, F. Leclercq, R. A. Marino, M. Maseda, A. Monreal-Ibero, T. Nanayakkara, J. Richard, R. Saust, M. Steinmetz \& M. Wendt

Correction to: Nature https://doi.org/10.1038/s41586-018-0564-6, published online 01 October 2018.

In this Letter, author M. Akhlaghi should be associated with affiliation (2) (Université Lyon, Université Lyon 1, ENS de Lyon, CNRS, Centre de Recherche Astrophysique de Lyon, Saint-Genis-Laval, France) instead of affiliation (3) (Leiden Observatory, Leiden University, Leiden, The Netherlands). This error has been corrected online. 